\title{
Sentimental Analysis from Video
}

\author{
Nikita Gavhane $^{1}$, Sayali Kolte ${ }^{2}$, Smita Botre ${ }^{3}$, Prof. Avinash Palave ${ }^{4}$ \\ Student, Comp Dept, KJEI'S Trinity College of Engineering and Research, Pisoli, Pune, India ${ }^{1,2,3}$ \\ Guide, Comp Dept, KJEI’S Trinity College Of Engineering and Research, Pisoli, Pune, India ${ }^{4}$
}

\begin{abstract}
Communication through voice is one of the main components of affective computing in human-computer interaction. [5]In this type of interaction, properly comprehending the meanings of the words or the linguistic category and recognizing the emotion included in the speech is essential for enhancing the performance. In order to model the emotional state, the speech waves are utilized, which bear signals standing for emotions such as boredom, fear, joy and sadness etc...So we can find different speech signals of each subject. The most significant features that transfer the variations in the tone are classified into pitch and intensity categories. We can use, eleven features, namely, pitch, intensity, the first four formants and their bandwidths and standard deviation, are extracted. The proposed method first digitizes the signal to extract the required properties. According to emotional Prosody studies, the tone of every person's voice can be characterized by its pitch, loudness or intensity, timbre, speech rate and pauses, whose changes convey different information from the speaker to the listener.[6]
\end{abstract}

Keywords: Speaker recognition, vocal emotion recognition, sentimental analysis, Emotion prediction, Text mining

\section{INTRODUCTION}

In this paper a large proportion of these videos, people depict their opinions about products, movies, social issues, political issues, etc. The capability of detecting the sentiment of the speaker in the video can serve two basic functions: (i) it can enhance the retrieval of the particular video in question, thereby, increasing its utility, and (ii) the combined sentiment of a large number of videos on a similar topic can help in establishing the general sentiment. Sentiment analysis is extracting emotions from a piece of text for a given video. Sentiment analysis is known as emotion extraction or opinion mining. This is a very popular field of in text mining. The basic idea is to find the separation of the text and classify it into positive, negative or neutral. It helps in human decision making. To perform sentiment analysis, one has to perform various tasks like subjectivity detection, sentiment classification, aspect term extraction, feature extraction etc. This paper presents the survey of main approaches used for sentiment classification It is important to note that automatic sentiment detection using text is a mature area of research, and significant attention has been given to product reviews, we focus our attention on dual sentiment detection in videos based on audio and text analysis. Feature extraction is the important part to extract the characteristic of the emotional state of speech.[2]

\section{LITERATURE SURVEY}

"Normal-to-shouted speech spectral mapping for speaker recognition undervocal effort mismatch."[1]

Speaker recognition performance degrades substantially in case of vocal effort mismatch (e.g. shouted vs. normal speech) between test and enrollment utterances. Such a mismatch is often encountered, for example, in forensic speaker recognition. This paper introduces a novel spectral mapping method which, when employed jointly with a statistical mapping technique, converts the Mel-frequency band energies of normal speech towards their counterparts in shouted speech. The aim is to obtain more robust performance in speaker recognition by tackling vocal effort mismatch between enrollment and test utterances. The processing is performed on the speech signal before feature extraction. The proposed approach was evaluated by testing the performance of a state-of-the-art i-vector-based speaker recognition system with and without applying the spectral mapping processing to the enrollment data. The results show that preprocessing with the proposed approach results in considerable improvement in correct identification rates.

"A Study of Support Vector Machines for Emotional Speech Recognition.”[2]

In this paper, efficiency comparison of Support Vector Machines (SVM) and Binary Support Vector Machines (BSVM) techniques in utterance-based emotion recognition is studied. Acoustic features including energy, Mel-frequency campestral coefficients (MFCC), Perceptual linear predictive (PLP), Filter bank (FBANK), pitch, their first and second derivatives are used as frame-based features. Four basic emotions including anger, happiness, neutral and sadness in Interactive Emotional Dyadic Motion Capture (IEMOCAP) database are selected for training and evaluating in our experiments. The best accuracy of emotional speech recognition is $58.40 \%$ in average from SVM with polynomial 


\section{International Journal of Advanced Research in Computer and Communication Engineering}

Vol. 7, Issue 11, November 2018

kernel. Energy features combination with FBANK, pitch and their first and second derivatives features are the most suitable for computing utterance feature. Binary Support Vector Machines (BSVM) techniques show accuracy improvement in some emotions, such as sadness and happiness emotion.

“Learning utterance-level representations for speech emotion and age/gender recognition.”[3]

Accurately recognizing speaker emotion and age/gender from speech can provide better user experience for many spoken dialogue systems. In this study, we propose to use deep neural networks (DNNs) to encode each utterance into a fixed-length vector by pooling the activations of the last hidden layer over time. The feature encoding process is designed to be jointly trained with the utterance-level classifier for better classification. A kernel extreme learning machine (ELM) is further trained on the encoded vectors for better utterance-level classification. Experiments on a Mandarin dataset demonstrate the effectiveness of our proposed methods on speech emotion and age/gender recognition tasks.

"Biologically inspired speech emotion recognition."[4]

In Conventional feature-based classification methods do not apply automatic recognition of speech emotions, mostly because the precise set of spectral and prosodic features that is required to identify the emotional state of a speaker has not been determined yet. This paper presents a method that operates directly on the speech signal, thus avoiding the problematic step of feature extraction. Furthermore, this method com-bines the strengths of the classical source-filter model of human speech production with those of the recently introduced liquid state machine (LSM), a biologicallyinspired spiking neural network (SNN). The source and vocal tract components of the speech signal are first separated and converted into perceptually relevant spectral representations. These representations are then processed separately by two reservoirs of neurons. The output of each reservoir is reduced in dimensionality and fed to a final classifier. This method is shown to provide very good classification performance on the Berlin Database of Emotional Speech. This seems a very promising framework for solving efficiently many other problems in speech processing.

\section{PROPOSE SYSTEM}

We focus on videos because the nature of speech in these videos is more natural and spontaneous which makes automatic sentiment processing challenging. In Particular, automatic speech recognition (ASR) of natural audio streams and text spoke in audio is difficult and the resulting transcripts are not very accurate. The difficulty stems from a variety of factors including (i) noisy audio due to non-ideal recording conditions, (ii) foreign accents, (iii) spontaneous speech production, and (iv)diverse range of topics. Our approach towards sentiment extraction uses two main systems, namely, automatic speech recognition (ASR) system and text-based sentiment extraction system. For text based sentiment extraction, we propose a new method that uses part-of-speech tagging to extract text features and Maximum Entropy modelling to predict the polarity of the sentiments (positive or negative) using the text features.

\section{SYSTEM ARCHITECTURE}

Following diagram is our system's architecture diagram:

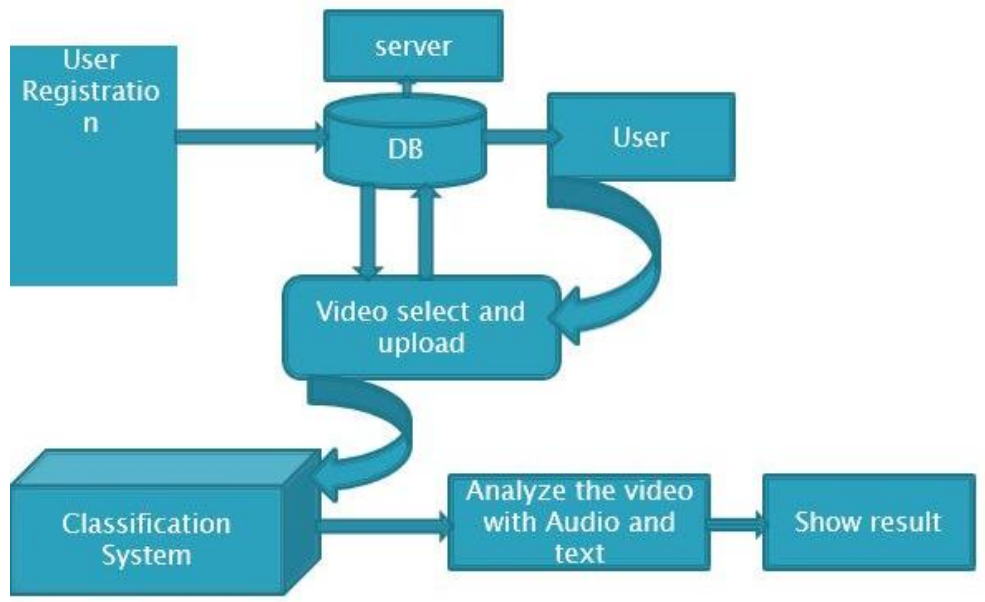

Figure 1: system architecture

In system architecture user can upload the video in application. After that analyzed the video with Audio and text. And after that user get result. 


\section{IJARCCE}

International Journal of Advanced Research in Computer and Communication Engineering

Vol. 7, Issue 11, November 2018

\section{SENTIMENTAL ANALYSIS}

Sentimental analysis is process of extracting emotions from given video. Sentiment level can be classified in below. प्र Sentence Level:-Each sentence is analyzed separately and classified as negative, positive or objective.

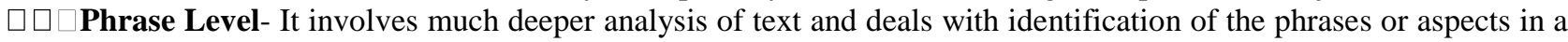
sentence and analyzing the phrases and classify them as positive, negative or objective.

3. Document Level- In it the whole document is given a single polarity positive, negative or objective.

\section{METHODOLOGIES}

For a text based extraction, we propose a new method that uses POS (part-of-speech) tagging to extract text features and Maximum Entropy modelling to predict the polarity of the sentiments (positive or negative) using the text features. An important feature of our method is the ability to identify the individual contributions of the text features towards sentiment estimation. We evaluate the proposed sentiment estimation on both publically available text databases and videos. On the text datasets, this provides us with the capability of identifying key words/phrases within the video that carry important information. By indexing these key words/phrases, retrieval systems can enhance the ability of users to search for relevant information. Sentiment analysis deals with identifying and classifying opinions or sentiments expressed in source text. Sentiment analysis is compared to general sentiment analysis due to the presence of emotions.

\section{CONCLUSION}

This paper investigated the prediction of the next reactions from emotional vocal signals based on the recognition of emotions, using different categories of classifiers. In this research, the trained dataset was used for modeling the emotional state. This database includes speech files in the wave format. The leadership, anger, disagree, self-control emotions were chosen. Eleven spectral features, namely, pitch, intensity, the first four formants and their bandwidths and standard deviation, were extracted.

\section{REFERRENCES}

[1]. "Normal-to-shouted speech spectral mapping for speaker recognition undervocal effort mismatch.” Ram'irez L'opez, Rahim Saeidi, Lauri Juvela, Paavo Alku978-1-5090-4117-6/17/\$31.00 @2017 IEEE.

[2]. "A Study of Support Vector Machines for Emotional Speech Recognition.” Nattapong Kurpukdee *†, Sawit Kasuriya $\dagger$, Vataya Chunwijitra $\dagger$, Chai Wutiwiwatchai $\uparrow$ and Poonlap Lamsrichan *2017 8th International Conference of Information and Communication Technology for Embedded Systems.

[3]. “Learning utterance-level representations for speech emotion and age/gender recognition.”Zhong-Qiu Wang1 and Ivan Tashev 2978-1-50904117-6/17/\$31.00 @2017 IEEEICASSP 2017.

[4]. "Biologically inspired speech emotion recognition." Reza Lotjidereshgi, Philippe Gournay978-1-5090-4117-6/ 17/\$3 1.00 @2017 IEEEICASSP2017.

[5]. "Speech-based Emotion Recognition and Next

[6]. Reaction Prediction.” Fatemeh NorooziNeda Akrami, Gholamreza Anbarjafari 978-1-5090-6494-6/17/\$31.00 _c 2017 IEEE

[7]. Sentiment Analysis of Speech Aishwarya Murarka1, Kajal Shivarkar2, Sneha3, Vani Gupta4,Prof.Lata Sankpal5ISSN (Online) 2278-1021 ISSN (Print) 2319 5940DOI 10.17148/IJARCCE.2017.61137

[8]. G. Shani and A. Gunawardana, "Evaluating recommendation systems," Recommender systems handbook, Springer, 2011 , pp. $257-297$.

[9]. D.N. Chin, "Empirical evaluation of user models and user-adapted systems," User modeling and user-adapted interaction, vol. 11, 2001 , pp. 181-194.

[10]. P. Brusilovsky and E. Millán, "User models for adaptive hypermedia and adaptive educational systems," The adaptive web, 2007 , pp. 3-53.

[11]. S. Lam, D. Frankowski, and J. Riedl, "Do you trust your recommendations? An exploration of security and privacy issues in recommender systems," Emerging Trends in Information and Communication Security, 2006, pp. 14-29. 